\title{
Prevention of post-mastectomy neuropathic pain with memantine: study protocol for a randomized controlled trial
}

\author{
Gisèle Pickering ${ }^{1,2,3^{*}}$, Véronique Morel ${ }^{1,2,3}$, Dominique Joly ${ }^{4}$, Christine Villatte ${ }^{4}$, Delphine Roux ${ }^{3}$, \\ Claude Dubray ${ }^{1,2,3}$ and Bruno Pereira ${ }^{5}$
}

\begin{abstract}
Background: N-methyl-D-aspartate receptor antagonists are potential therapies for neuropathic pain, and memantine has a good tolerance profile. A preclinical study recently reported that presurgery memantine may prevent neuropathic pain development and cognition dysfunction. Considering the high prevalence of breast cancer and of post-mastectomy neuropathic pain, a clinical trial is carried out to evaluate if memantine may prevent neuropathic pain development and maintain cognitive function and quality of life in cancer patients.

Methods/Design: A randomized clinical trial (NCT01536314) includes 40 women with breast cancer undergoing mastectomy at the Oncology Hospital, Clermont-Ferrand, France. Memantine (5 to 20 mg/day; $n=20$ ) or placebo $(n=20)$ is administered for 4 weeks starting 2 weeks before surgery. Intensity of pain, cognitive function, quality of life and of sleep, anxiety and depression are evaluated with questionnaires. The primary endpoint is pain intensity on a 0 to 10) numerical scale at 3 months post-mastectomy. Data analysis is performed using mixed models and the tests are two-sided, with a type I error set at $a=0.05$.

Discussion: The hypothesis of this translational approach is to confirm in patients the beneficial prophylactic effect of memantine observed in animals. Such a protective action of memantine against neuropathic pain and cognitive dysfunction would greatly improve the quality of life of cancer patients.
\end{abstract}

Trial registration: ClinicalTrials.gov: NCT01536314 on 16 February 2012

Keywords: Memantine, NMDA receptor, Breast cancer, Mastectomy, Chemotherapy, Neuropathic pain

\section{Background}

Medical treatment of neuropathic pain (NP) is still far from being satisfactory, with less than half the patients achieving significant benefit with any pharmacological drug [1]. Several therapies have been developed for the treatment of NP but these methods are not equally effective for all NP patients. N-methyl-D-aspartate receptor (NMDAR) antagonists such as ketamine, memantine or dextromethorphan are potential drugs for NP alleviation [2]. Evidence suggests that NMDAR within the dorsal horn plays an important role in both inflammation and

\footnotetext{
*Correspondence: gisele.pickering@udamail.fr

'Clermont Université, Université d'Auvergne, Pharmacologie Fondamentale

et Clinique de la Douleur, Laboratoire de Pharmacologie, Facultés de

Médecine/Pharmacie, F-63000 Clermont-Ferrand, France

${ }^{2}$ Inserm, U1 107 Neuro-Dol, F-63001 Clermont-Ferrand, France

Full list of author information is available at the end of the article
}

nerve injury-induced central sensitization [3]. Activation of NMDAR is associated with abnormalities in the sensory (peripheral and central) system, resulting in neuronal excitation and abnormal pain manifestations (spontaneous pain, allodynia, hyperalgesia) [4]. Blocking these receptors by antagonists leads to a reduction in pain [5]. A recent review of the literature including 28 randomized clinical trials [2] emphasizes the heterogeneity of doses used, the diversity of pathologies generating neuropathic pain (postherpetic, post-amputation, diabetes and so forth) and highlights the need to develop clinical trials of good methodological quality with NMDA antagonists. NMDAR antagonists, such as ketamine [6,7], are prescribed after therapeutic failure with classical treatment but these drugs have severe adverse events that limit their clinical use [8]. Another NMDAR antagonist, memantine, prescribed in 
Alzheimer's disease to maintain cognitive function, has minimal side-effects at doses within the therapeutic range, probably because of its specific mechanism of action as it is an uncompetitive antagonist with moderate affinity, strong voltage-dependency and rapid unblocking kinetics [9-11]. Concerning NP alleviation, memantine shows controversial results in human studies [6,12-15]. We recently demonstrated for the first time in an animal surgical NP model, that memantine prevents the development of NP symptoms and the impairment of spatial memory [16]. With a translational approach, we present a clinical study where memantine (versus placebo) is administered 2 weeks before and 2 weeks after mastectomy in 40 women suffering from breast cancer. Confirmation of preclinical results in this clinical study would constitute a major step for NP prevention by memantine and maintenance of cognition and quality of life in these vulnerable patients.

\section{Methods/Design}

We are conducting a randomized, placebo-controlled clinical trial in the Oncology Hospital, ClermontFerrand, France, in 40 women undergoing total mastectomy for breast cancer. The study has been approved in December 2011 by the regional Ethics committee (CPP Sud-Est, France, number AU917) and registered on 16 February 2012 at "http://www.clinicaltrials.gov" (NCT01536314). Women provide written informed consent prior to their participation in the study during their anesthesiology visit. After baseline assessments (day (D) ${ }_{-15}$ ) of pain intensity, cognition, quality of life and quality of sleep questionnaires, participants are randomized into two parallel groups: memantine $(n=20)$ or placebo $(n=20)$. Memantine or placebo (lactose) is given orally for 4 weeks starting 2 weeks before surgery. Memantine is given in increasing doses: $5 \mathrm{mg} /$ day for 3 days; $10 \mathrm{mg} /$ day for 3 days; $15 \mathrm{mg} /$ day for 3 days and $20 \mathrm{mg} /$ day for 5 days. Endpoints are reassessed 15 days $\left(\mathrm{D}_{0+15}\right)$, 3 months ( $\mathrm{D}_{0}+3$ months) and 6 months $\left(\mathrm{D}_{0}+6\right.$ months $)$ post-mastectomy. In order to maintain a good compliance and to verify that women do not develop adverse events, patients are called once a week by phone. A booklet for monitoring is completed daily by the patient for 6 months from the day of surgery. Detailed information on the present study is summarized in Figure 1.

\section{Eligibility}

\section{Inclusion criteria}

Women are eligible for this study if they are at least 18 years old, with a diagnosis of breast cancer, programmed for mastectomy with or without axillary dissection, able to understand and willing to follow the study protocol.

\section{Exclusion criteria}

Exclusion criteria comprise contraindications for memantine and hypertension, severe cardiac insufficiency or diabetes (Type I and II), alcohol addiction and treatment with specific drugs (amantadine, ketamine, dextromethorphan, L-Dopa, dopaminergic, anticholinergic agonists, barbituric, neuroleptic, IMAO, antispastic agents, dantrolen or baclofen, phenytoin, cimetidine, ranitidine, procainamide, quinidine, quinine, nicotine, hydrochlorothiazide, warfarin). Childbearing age, no use of effective contraceptive method, pregnancy or lactation, involvement in another clinical trial and inability to comply with the requirements of protocol are other exclusion criteria.

\section{Objectives}

The primary objective of this study is to evaluate if memantine administered before and after mastectomy may prevent pain development at 3 months postmastectomy when compared to the placebo group. The endpoint at 3 months was chosen because chronic pain is usually defined as pain lasting longer than 2 to 3 months $[17,18]$, and 6 months was included as a secondary endpoint.

The secondary objectives are to estimate at 3 and 6 months post-mastectomy the pain intensity, the analgesic concomitant medications, the impact of treatment (memantine/placebo) on cognitive function, quality of life, sleep, anxiety and depression, the impact of cancer chemotherapy-induced pain and cognitive impairment and the prevalence of phantom breast experience.

\section{Definition of endpoints and outcome measures Primary endpoint}

The primary endpoint is the pain intensity evaluation by numerical scale in memantine and placebo groups at 3 months post-mastectomy. The scale ranges from 0 (no pain) to 10 (maximal tolerable pain).

\section{Secondary endpoints}

Secondary endpoints are the evaluation of pain at the screening visit, at 2 weeks, 3 months and 6 months after mastectomy (numerical scale, Brief pain Inventory, McGill pain questionnaire), neuropathic pain (Neuropathic Pain in 4 questions, Neuropathic Pain Symptom Inventory), cognition (Trail Making Test, Digit Symbol Substitution Test), sleep (Leeds sleep questionnaire), quality of life (Short-Form-36), and anxiety and depression (Hospital Anxiety and Depression scale). The summary of the different evaluations for a patient is reported in Table 1.

Brief Pain Inventory This self-administered questionnaire provides information on the intensity of pain, along with the degree to which the pain interferes with the everyday functioning of life including: mood, walking, 


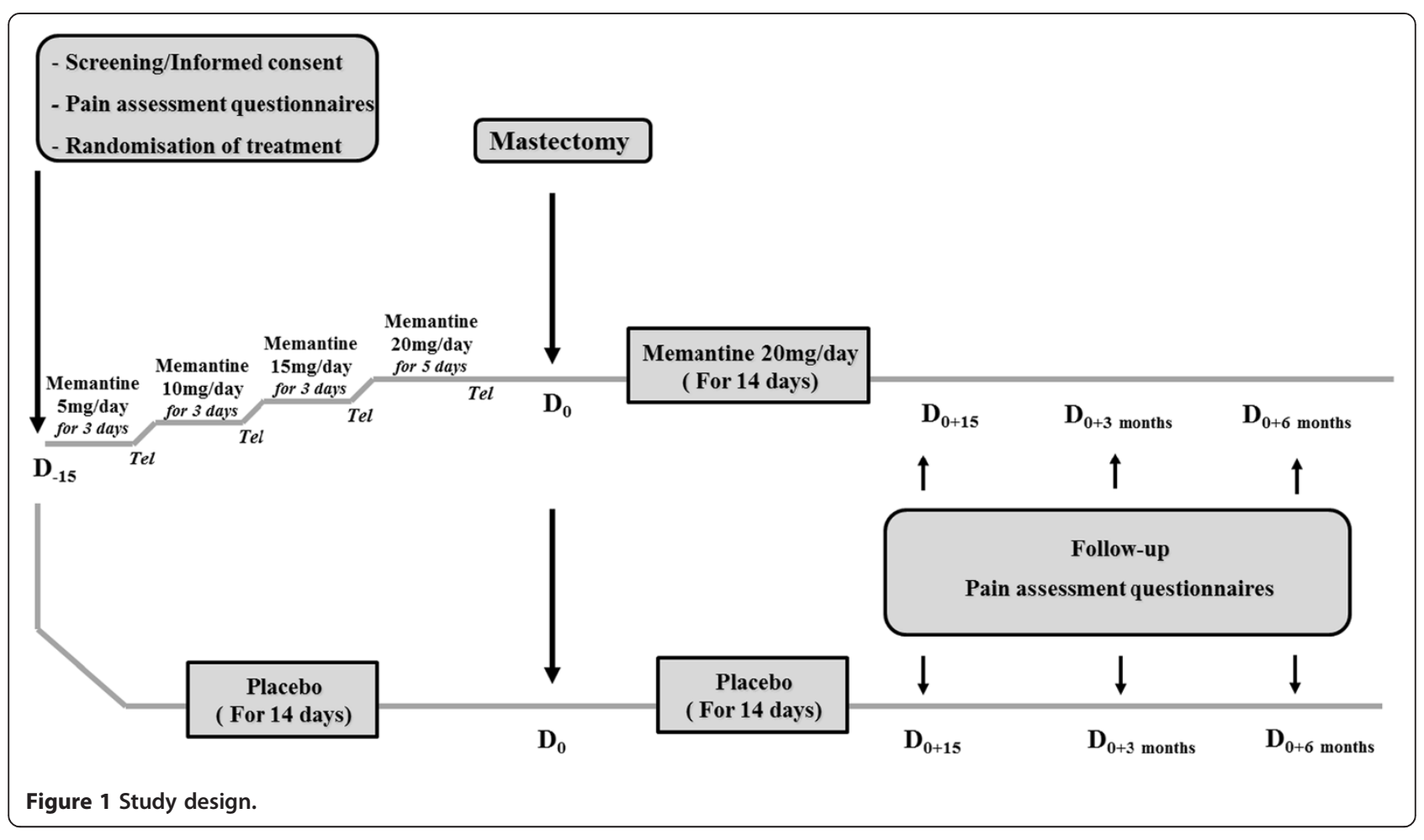

Table 1 Summary of evaluation for a patient

\begin{tabular}{lcccccc}
\hline Visit & $\begin{array}{c}\text { Screening/informed } \\
\text { consent }\end{array}$ & $\begin{array}{c}\text { Surgery } \\
\text { (mastectomy) }\end{array}$ & Hospital stay & Follow-up & Follow-up & Follow-up \\
\hline Day of visit & $D_{0-15}$ & $D_{0}$ & $D_{0}$ to $D_{0+15}$ & $D_{0+15}$ & $D_{0+3}$ months & $D_{0+6}$ months \\
& & Filling of questionnaires & & &
\end{tabular}

1- Pain

Numerical Scale

DN4

NPSI

$\mathrm{BPI}$

McGill pain questionnaire

\section{2- Cognition}

Trail Making Test

Digit Symbol Substitution Test

\section{3- Quality of life}

SF-36

\section{4- Sleep}

Leeds questionnaire

\section{5- Anxiety/Depression}

HAD scale

\section{6- Concomitant medication recording}

Treatments (analgesics, antidepressants, etc.)

$\begin{array}{lllllll}+ & - & + & + & + & + \\ + & - & - & + & + & + \\ - & - & - & + & + & + \\ - & - & - & - & + & + \\ - & - & - & - & + & +\end{array}$

+, Questionnaire performed; -, questionnaire not carried out; BPI, Brief Pain Inventory; DN4, Neuropathic Pain in 4 questions; HAD scale, Hospital Anxiety and Depression Scale; NPSI, Neuropathic Pain Symptom Inventory; SF-36, Short-Form-36. 
general activity, relations with others, sleep, enjoyment of life [19].

McGill pain questionnaire This questionnaire allows the patient to describe pain experienced during the last 48 hours [20]. It has fifty eight qualifiers divided into sixteen items (A to P). Each qualifier is rated from 0 to 4 , where 0 = absent, 1 = low, $2=$ moderate, $3=$ strong, $4=$ very strong. The score is divided between two subclasses: sensory subclass (items A to I) and emotional subclass (items J to P).

Neuropathic pain: "Neuropathic Pain in 4 questions" Neuropathic pain in four questions is a clinical tool for the diagnosis of neuropathic pain [21]. This questionnaire has four questions divided into 10 items related to the interview (that is, symptoms) and to the sensory examination (that is, signs). The investigator asks and examines the patient and notes a response "no" or "yes" for each item: "yes" is scored as "1" and "no" is scored as " 0 ". The sum of scores gives the total score of the patient (out of 10). Neuropathic pain in four questions is considered as positive if the patient obtains a score of $4 / 10$.

Neuropathic Pain Symptom Inventory Neuropathic Pain Symptom Inventory is a self-questionnaire and includes 10 pain descriptors [22]. Intensity is rated on 0 to 10 numerical scales and two temporal items are designed to assess spontaneous ongoing pain duration and the number of pain paroxysms over 24 hours. This questionnaire discriminates five distinct clinically relevant dimensions: spontaneous burning pain, spontaneous deep pain, paroxysmal pain, evoked pain, and paresthesia/ dysesthesia.

Trail Making Test This non-verbal cognitive test assesses the ability of speed, executive functions, attention, concentration, and visual perceptual speed $[23,24]$. The test takes place in two parts: in Part A, circles are numbered from 1 to 25 and the patient must connect with lines the numbers in ascending order (1-2-3-4, and so forth); in Part B, the circles contain numbers from 1 to 13 and letters from $\mathrm{A}$ to $\mathrm{L}$, the patient must connect the circles with lines but alternating numbers and letters (1A-2B -3C, and so forth). The patient must connect the circles as quickly as possible for both parts of the test, without lifting the pen from the paper. The Trail Making Test B additionally provides an estimate of mental flexibility.

Digit symbol substitution test The digit symbol substitution test is a neuropsychological, non-verbal test, which assesses cognitive deficit and brain damage associated with aging and/or depression [25]. It also evaluates learning ability, concentration and attention. It consists of combining pairs of symbols and numbers as quickly as possible and the score is the correct number of symbols in the time allowed (for example, 90 or 120 seconds).

Short-Form 36 The Short-Form-36 is a questionnaire evaluating the quality of life of patients $[26,27]$. It is a multidimensional scale that assesses the health and quality of life. This scale can be performed in self- or heteroquestionnaire with 36 items including nine dimensions: physical function, role physical, bodily pain, general health, vitality, social functioning, role emotional, mental health and health thinking.

Leeds sleep questionnaire The Leeds sleep evaluation $[28,29]$ questionnaire is a standardized self-administered questionnaire composed of ten visual analogue scales that relate to four aspects of sleep efficiency: quality of sleep, getting to sleep (visual scales 1, 2 and 3); sleep quality (visual scales 4 and 5); awakening from sleep (visual scales 6, 7 and 8); and behavior following wakefulness (visual scales 9 and 10).

Hospital Anxiety and Depression scale (HAD) The Hospital Anxiety and Depression scale is a selfadministered questionnaire in 14 items completed by the patient [30]. It is used to determine the levels of anxiety and depression. Seven of the items relate to anxiety and seven relate to depression.

\section{Randomization, allocation concealment and blinding}

Women with breast cancer are informed by their anesthetist 2 to 3 weeks before mastectomy. On the day of the visit, inclusion and exclusion criteria are verified and written informed consent is obtained by the physician. After clinical examination and pain evaluation, the patient fills in the questionnaires. A clinical nurse independent from the protocol obtains the randomization number from the hospital pharmacy and the patient is then randomized in the memantine or placebo group. Treatment allocation follows a predetermined randomization list and is generated using random blocks. Memantine and placebo treatments are packed in similar blisters covered with an identical label indicating batch number, expiry date and sponsor code with no indication of the name of the drug. The nurse gives the treatment to the patient in an independent room after questionnaires have been filled. In order to maintain blinding, the consent physician (who evaluates pain, the main endpoint of the study) cannot guess allocation at any time and does not see the patient again before they leave hospital. 


\section{Sample size}

The number of subjects required is 40 chronic pain patients (20 in each group). The minimum $\delta$ difference in numerical scale pain between memantine and placebo groups at 3 months is estimated at 1.6 and $\sigma$ standard deviation at 1.5 , estimated from published data of the literature [31,32], with $\alpha=0.05$ two-sided situation and $\beta=0.10$.

\section{Statistical analysis}

Statistical analyses will be performed with Stata software (version 13, StataCorp, College Station, USA). Concerning the primary objective, comparison between the randomized groups will be performed using an analysis of covariance with baseline score as a covariate [33]. The correlation between baseline and follow-up scores was also proposed. For other secondary parameters, the comparisons between the randomized groups will be performed using the Student test or the Mann-Whitney test (if the conditions for validity of the Student test are not met, normality will be verified by the Shapiro-Wilk and homoscedasticity by the Fisher-Snedecor test). To study the evolution of the main endpoint (numerical scale pain), data analyses will be performed using mixed models which allow us to consider, on the one hand, time, group and interaction time versus group as fixed effects, and, on the other hand, the within- and betweensubject variability in order to visualise the assumption of a difference at 2 months between the two randomized groups that increases up to 3 months and stabilizes at 6 months. Residual normality will be checked for all considered models. When appropriate, anticancer chemotherapy (yes/no) will be studied as a fixed effect in these models before considering subgroup analyses. The comparison between the treatment groups will be performed systematically: (1) without adjustment; and (2) by adjusting other factors whose repartition could be, despite the randomization, unbalanced between the treatment groups. The tests will be two-sided, with a type I error set at $\alpha=$ 0.05 . A sensitivity analysis of missing data will be performed to ensure the pertinence of the longitudinal data (MAR (Missing at random) or MCAR (Missing completely at random)). In order to assess the problem caused by missing longitudinal data at 6 months, estimation methods developed by Verbeke and colleagues [34] will be proposed.

\section{Discussion}

NP is difficult to treat and NMDAR antagonists, such as ketamine, dextromethorphan or memantine [5,6,35], are potential drugs for persistent pain. Memantine has shown its efficacy in some studies [15,36] and presents the advantage of having less adverse effects than ketamine [8-11]. We have shown in a surgical pain model that memantine prevents NP symptoms such as tactile allodynia and mechanical hyperalgesia when administered a few days before surgery [16]. These findings needed to be confirmed in patients during the postoperative period. Mastectomy is known to generate NP in $23 \%$ patients at 3 months post-surgery [37], $42 \%$ at 5 years [38] and 37\% at 9 years post-mastectomy [39]. Cancer patients undergoing surgery may also develop NP associated with cancer chemotherapy. It is well known that $25 \%$ to $50 \%$ of patients treated with chemotherapeutic agents such as taxane, vinca alkaloid and platinum classes develop peripheral neuropathy syndrome. Patients with chemotherapy-induced NP display a set of neuropathic pain symptoms that are characterized by stinging, tingling, numbness, changes in sensitivity, burning sensations or electric shocks [40]. The incidence of chemotherapy-induced NP depends on the type of chemotherapeutic agents, the dose administered and the cumulative dose.

This trial of prophylactic memantine given long before surgery in cancer patients aims to evaluate if memantine may diminish overall post-surgery pain and prevent NP development. It aims also to evaluate the concomitant impact on cognition and quality of life. We showed in animals that pre-surgery memantine prevents cognition impairment [16]. Breast cancer as well as chronic pain induced by chemotherapy may be associated with cognitive dysfunction [41] and impairment of health-related quality of life, quality of sleep, anxiety and/or depression [42]. Indeed, cognitive impairment may be due to the diagnosis of the pathology, to surgery [41] and also to chemotherapy, as cognitive deficit is described in 20$30 \%$ to $75 \%$ of women having chemotherapy-induced NP $[43,44]$.

Given that mastectomy with or without chemotherapy can induce NP and cognitive and emotional impairment, these complications represent a real burden for women with breast cancer, and global care is a priority to improve their well-being. If our study confirms preclinical results, memantine given before mastectomy could be a new prophylactic strategy to counteract NP development. It would provide a preventive therapeutic innovation to decrease the incidence of post-operative and chemotherapy-induced NP, cognitive impairment and quality of life impairment and comorbidities that generally accompany breast cancer pathology.

\section{Trial status}

Recruitment started in March 2012.

Abbreviations

NMDAR: N-methyl-D-aspartate receptor; NP: neuropathic pain.

\section{Competing interests}

The authors declare that they have no competing interests. 


\section{Authors' contributions}

GP is the overall study principal investigator; she participated in the conception and study design and contributed to the writing of the study protocol and the drafting and editing of this manuscript. DJ, CV, DR, CD and $\mathrm{BP}$ all participated in the study design. BP, DR and CD contributed to the writing of the study protocol. BP carried out all statistical calculations and wrote the statistical paragraph in the study protocol. He contributed with GP and VM to the drafting and editing of this manuscript and the interpretation of data for the work. All authors read and approved the final manuscript.

\section{Acknowledgements}

We thank the "Act against pain: APICIL" Foundation for financial support for this study.

\section{Funding}

"APICIL" Foundation.

\section{Author details}

${ }^{1}$ Clermont Université, Université d'Auvergne, Pharmacologie Fondamentale et Clinique de la Douleur, Laboratoire de Pharmacologie, Facultés de Médecine/Pharmacie, F-63000 Clermont-Ferrand, France. ${ }^{2}$ Inserm, U1107 Neuro-Dol, F-63001 Clermont-Ferrand, France. ${ }^{3} \mathrm{CHU}$ Clermont-Ferrand, Inserm CIC 1405, Centre de Pharmacologie Clinique, F-63003 Clermont-Ferrand, France. ${ }^{4} \mathrm{CHU}$ Clermont-Ferrand, Centre Jean Perrin, Centre de Lutte contre le Cancer, 58 rue Montalembert, F-63000 Clermont-Ferrand, France. ${ }^{5} \mathrm{CHU}$ de Clermont-Ferrand, Délégation Recherche Clinique \& Innovation - Villa annexe IFSI, 58 Rue Montalembert, F-63003

Clermont-Ferrand cedex, France.

Received: 6 March 2014 Accepted: 6 August 2014 Published: 20 August 2014

\section{References}

1. Finnerup NB, Otto M, McQuay HJ, Jensen TS, Sindrup SH: Algorythm for neuropathic pain treatment: an evidence based proposal. Pain 2005 118:239-305.

2. Collins S, Sigtermans MJ, Dahan A, Zuurmond WW, Perez RS: NMDA receptor antagonists for the treatment of neuropathic pain. Pain Med 2010, 11:1726-1742

3. Bleakman D, Alt A, Nisenbaum ES: Glutamate receptors and pain Semin Cell Dev Biol 2006, 17:592-604.

4. Petrenko AB, Yamakura $T$, Baba H, Shimoji $K$ : The role of N-methyl-D-aspartate (NMDA) receptors in pain: a review. Anesth Analg 2003, 97:1108-1116.

5. Zhou HY, Chen SR, Pan HL: Targeting N-methyl-D-aspartate receptors for treatment of neuropathic pain. Expert Rev Clin Pharmacol 2011, 4:379-388.

6. Sang CN, Booher S, Gilron I, Parada S, Max MB: Dextromethorphan and memantine in painful diabetic neuropathy and postherpetic neuralgia: efficacy and dose-response trials. Anesthesiology 2002, 96:1053-1061.

7. Correll GE, Maleki J, Gracely EJ, Muir JJ, Harbut RE: Subanesthetic ketamine infusion therapy: a retrospective analysis of a novel therapeutic approach to complex regional pain syndrome. Pain Med 2004, 5:263-275.

8. Cvrcek P: Side effects of ketamine in the long-term treatment of neuropathic pain. Pain Med 2008, 9:253-257.

9. Parsons CG, Danysz W, Bartmann A, Spielmanns P, Frankiewicz T, Hesselink M, Eilbacher B, Quack G: Amino-alkyl-cyclohexanes are novel uncompetitive NMDA receptor antagonists with strong voltagedependency and fast blocking kinetics: in vitro and in vivo characterization. Neuropharmacology 1999, 38:85-108.

10. Parsons CG, Danysz W, Quack G: Memantine is a clinically well tolerated NMDA receptor antagonist - a review of preclinical data. Neuropharmacology 1999, 38:735-767.

11. Jonhson JW, Kotermanski SE: Mechanism of action of memantine. Curr Opin Pharmacol 2006, 6:61-67.

12. Eisenberg E, Kleiser A, Dortort A, Haim T, Yarnitsky D: The NMDA (Nmethyl-D-aspartate) receptor antagonist memantine in the treatment of postherpetic neuralgia: a double-blind, placebo-controlled study. Eur J Pain 1998, 2:321-327.

13. Nikolajsen L, Gottrup H, Anders GD, Jensen TS: Memantine (a N-methyl-Daspartate receptor antagonist) in the treatment of neuropathic pain after amputation or surgery: a randomized, double-blind, cross-over study. Anesth Analg 2000, 91:960-966.
14. Maier C, Dertwinkel R, Mansourian N, Hosbach I, Schwenkreis P, Senne I, Skipka G, Zenz M, Tegenthoff M: Efficacy of the NMDA-receptor antagonist memantine in patients with chronic phantom limb pain-results of a randomized double-blinded, placebo-controlled trial. Pain 2003, 103:277-283.

15. Hackworth RJ, Tokarz KA, Fowler IM, Wallace SC, Stedje-Larsen ET: Profound pain reduction after induction of memantine treatment in two patients with severe phantom limb pain. Anesth Analg 2008, 107:1377-1379.

16. Morel V, Etienne M, Wattiez AS, Dupuis A, Privat AM, Chalus M, Eschalier A, Daulhac L, Pickering G: Memantine, a promising drug for the prevention of neuropathic pain in rat. Eur J Pharmacol 2013, 721:382-390.

17. Turk DC, Okifuji A: Pain terms and taxonomies. Bonica's Management of Pain 2001, 18-25.

18. Ravindran D: Chronic postsurgical pain: prevention and management. J Pain Palliat Care Pharmacother 2014, 28:51-53.

19. Cleeland CS, Ryan KM: Pain assessment: global use of the Brief Pain Inventory. Ann Acad Med Singapore 1994, 23:129-138.

20. Boureau F, Luu M, Doubrère JF: Comparative study of the validity of four French McGill Pain Questionnaire (MPQ) versions. Pain 1992, 50:59-65.

21. Bouhassira D, Attal N, Alchaar H, Boureau F, Brochet B, Bruxelle J, Cunin G, Fermanian J, Ginies P, Grun-Overdyking A, Jafari-Schluep H, Lantéri-Minet M, Laurent B, Mick G, Serrie A, Valade D, Vicaut E: Comparison of pain syndromes associated with nervous or somatic lesions and development of a new neuropathic pain diagnostic questionnaire (DN4). Pain 2005, 114:29-36.

22. Bouhassira D, Attal N, Fermanian J, Alchaar H, Gautron M, Masquelier E, Rostaing S, Lanteri-Minet M, Collin E, Grisart J, Boureau F: Development and validation of the Neuropathic Pain Symptom Inventory. Pain 2004, 108:248-257.

23. Reitan RM, Wolfson D: The Halstead-Reitan Neuropshychological Test Battery: Therapy and Clinical Interpretation. Tucson, AZ: Neuropsychological Press; 1985.

24. Tombaugh TN: Trail Making Test A and B: normative data stratified by age and education. Arch Clin Neuropsychol 2004, 19:203-214

25. Crowe SF, Benedict T, Enrico J, Mancuso N, Matthews C, Wallace J: Cognitive determinants of performance on the digit symbol-coding test, and the symbol search test of the wais-III, and the symbol digit modalities test: an analysis in a healthy sample. Aust Psychol 1999, 34:204-210.

26. Ware JE Jr, Sherbourne CD: The MOS 36-item short-form health survey (SF-36). I. Conceptual framework and item selection. Med Care 1992, 30:473-483

27. Leplège A, Ecosse E, Verdier A, Perneger TV: The French SF-36 Health Survey: translation, cultural adaptation and preliminary psychometric evaluation. J Clin Epidemiol 1998, 51:1013-1023.

28. Parrott AC, Hindmarch I: The Leeds sleep evaluation questionnaire in psychopharmacological investigations, a review. Psychopharmacology (Berl) 1980, 71:173-179.

29. Tarrasch R, Laudon M, Zisapel N: Cross-cultural validation of the Leeds sleep evaluation questionnaire (LSEQ) in insomnia patients. Hum Psychopharmacol 2003, 18:603-610.

30. Zigmond AS, Snaith RP: The hospital anxiety and depression scale. Acta Psychiatr Scand 1983, 67:361-370.

31. Pickering G, Pereira B, Dufour E, Soule S, Dubray C: Impaired modulation of pain in patients with postherpetic neuralgia. Pain Res Manag 2014, 19:e19-e23.

32. Van Seventer R, Bach FW, Toth CC, Serpell M, Temple J, Murphy TK, Nimour $M$ : Pregabalin in the treatment of post-traumatic peripheral neuropathic pain: a randomized double-blind trial. Eur J Neurol 2010, 17:1082-1089.

33. Vickers AJ, Altman DG: Statistics notes: analysing controlled trials with baseline and follow up measurements. BMJ 2001, 323:1123-1124.

34. Verbeke G, Fieuws S, Molenberghs G, Davidian MA: The analysis of multivariate longitudinal data: review. Stat Methods Med Res 2014, 23:42-59.

35. Jørum E, Warncke T, Stubhaug A: Cold allodynia and hyperalgesia in neuropathic pain: the effect of N-methyl-D-aspartate (NMDA) receptor antagonist ketamine-a double-blind, cross-over comparison with alfentanil and placebo. Pain 2003, 101:229-235.

36. Schley M, Topfner S, Wiech K, Schaller HE, Konrad CJ, Schmelz M, Birbaumer $\mathrm{N}$ : Continuous brachial plexus blockade in combination with the NMDA receptor antagonist memantine prevents phantom pain in acute traumatic upper limb amputees. Eur J Pain 2007, 11:299-308.

37. Bokhari FN, McMillan DE, McClement S, Daeninck PJ: Pilot study of a survey to indentify the prevalence of and risk factors for chronic neuropathic pain following breast cancer surgery. Oncol Nurs Forum 2012, 39:E141-E149.

38. Peuckmann V, Ekholm O, Rasmussen NK, Groenvold M, Christiansen P, Moller S, Eriksen J, Sjogren P: Chronic pain and other sequelae in long-term breast cancer survivors: nationwide survey in Denmark. Eur J Pain 2009, 13:478-485. 
39. Macdonald L, Bruce J, Scott NW, Smith WC, Chambers WA: Long-term follow-up of breast cancer survivors with post-mastectomy pain syndrome. Br J Cancer 2005, 92:225-230.

40. Golan-Vered Y, Pud D: Chemotherapy-induced neuropathic pain and its relation to cluster symptoms in breast cancer patients treated with paclitaxel. Pain Pract 2013, 13:46-52.

41. Hedayati E, Schedin A, Nyman H, Alinaghizadeh H, Albertsson M: The effects of breast cancer diagnosis and surgery on cognitive functions. Acta Oncol 2011, 50:1027-1036.

42. Reich M, Lesur A, Perdrizet-Chevallier C: Depression, quality of life and breast cancer: a review of the literature. Breast Cancer Res Treat 2008, 110:9-17.

43. Cimprich B, Reuter-Lorenz P, Nelson J, Clark PM, Therrien B, Normolle D, Berman MG, Hayes DF, Noll DC, Peltier S, Welsh RC: Prechemotherapy alterations in brain function in women with breast cancer. $J$ Clin Exp Neuropsychol 2010, 32:324-331.

44. Ahles TA, Saykin AJ, Furstenberg CT, Cole B, Mott LA, Skalla K, Whedon MB, Bivens S, Mitchell T, Greenberg ER, Silberfarb PM: Neuropsychologic impact of standard-dose systemic chemotherapy in long-term survivors of breast cancer and lymphoma. J Clin Oncol 2002, 20:485-493.

doi:10.1186/1745-6215-15-331

Cite this article as: Pickering et al:: Prevention of post-mastectomy neuropathic pain with memantine: study protocol for a randomized controlled trial. Trials 2014 15:331.

\section{Submit your next manuscript to BioMed Central and take full advantage of:}

- Convenient online submission

- Thorough peer review

- No space constraints or color figure charges

- Immediate publication on acceptance

- Inclusion in PubMed, CAS, Scopus and Google Scholar

- Research which is freely available for redistribution 